Dermatologische Zeitschrift. 1931;61:I-IV

\title{
Contents, Vol. 61, 1931
}

Inhaltsverzeichnis.

Or $1 / 8$ inalarbeiten.

$1 / 8 \quad$ Seit $\beta$

ßalog, Paul, Beiträge zur Frage des Granuloma teleangiektodes . . 31

Behrmann, K., siehe Bruck, C.

Bruck, C, und K. Behrmann, Die Meinicke-Klärungsreaktion im Vergleich zu derAktivkomplementbindung nach Bruck und der Original-

Wassermann-Reaktion 7

Fellner, M., Bemerkungen über psoriasiforme Salvarsan-(Syntharsan-)

Exantheme und Beeinflussung der morphologischen Gestaltung der

Psoriasis bei einem Luetiker durch spezifische Therapie $\quad 372$

Foerster, B., Zur Frage der Beziehungeu zwischen Alkalireserve und

Dermatosen 153

Grüneberg, Th, Fieberhafte Gelenkaffektionen bei Psoriasis.- .... 170

Hellerström, Sven, Experimentelle Untersuchungen über Lympho-

granuloma inguinale (L. i.) 395

Hofmann, Edmund, Über einige Fälle unspezifischer Genitalerkran-

kungen unter dem Bilde sekundär luetischer Papeln 10

Hoffmann, Erich, Zur 25jährigen Wiederkehr der Entdeckung der

Serodiagnostik der Syphilis 180

Jordan, A., und J. Staroff, Syphilis et mors 221

Kißmeyer, A., und Gerda Schultz, Untersuchungen über die sogenannten Antikutine und Prokutine im Serum von Sarkoidkranken 18

Lòhe, H., und H. Rosenfeld, Multiple Hautgangrän bei Periarteriitis nodosa. Ein Beitrag zur

Kenntnis der multiplen neurotischen Hautgangrän und der Hautveränderungen bei Periarteriitis

nodosa . . 299

Michael, Max, und Charlotteßadeff, KapillarmikroskopischeUnter

suchungen zur Konstitutionspathologie des Ekzems, insbesondere des

Gewerbeekzems 159

Müller, Alfred, Sensibilisierungsversuche mit Ursol 241

Photinos, Panagiotis B., Experimentelle Untersuchungen ûbcr die

syphilitische Lymphdrüsenerkrankung von Afl $1 / 8 \mathrm{n} \quad 27$

Beitrag zur Atiologie der Trichotillomanie 239

Serologische Untersuchungen bei gesunden u. syphilitisclien Kaninclien 400

Follicultis sycosíformis atrophicaus corporis. (Foliiculite dépilante des parties glabres d'Arnozan et de Dubreuilh) 321

Polland, ß., Multiples benignes Sarkoid bei einem Säugling 360

Badeff, Charlotte, siehe Michael, Max.

ßosenfeld, H., siehe Löhe, H. 
Schreiner, K., Über tertiäre Syphilis 365

Schultz, Gerda, siehe Kißmeyer, A.

Sluczewski, A., Über einen Fall von Dermatoneuromyositis 390

Staroff, J., siehe Jordan, A.

S t e r n , F r., Über den Einfluß von Hunger und Flüssigkeitseinschränkungen

auf die $\mathrm{K}-, \mathrm{Ca}-, \mathrm{Cl}-$ und Wasserverschiebungen in der bestrahíten

Kaninchenhaut $\quad 384$

IV Inhaltsverzeichnis.

Sßite

Strempel, Rud., Frühzeitiger Spirochätennachweis in skrotalen Impfherden von Kaninchen nach Einimpfung von Poplitealdrüsen latent

syphilitischer Kaninchen (Vorläufige Mitteilung) 1

- $\quad$ Die Serodiagnostik der Syphilis mittels der Meinicke-Klärungs- und

Kahn-Reaktion sowie der Ballungsreaktion nach Müller 143

Zelischtschewa, A. D., Vergleichende Schätzung der Antigene in der

Eeaktion Bordet-Gengou bei der Gonorrhoe 248

Zoon, J. J., Tierversuche mit Moniiia Fioccoi. (Nachtrag zur Arbeit

„Blastomycosis cutis usw.” aus Bd. 58 S. 356) 232

Gesellschaftsberichte.

Berliner Dermatologische Gesellschaft. Sitzung vom 9. XII. 1930 .... 41

Sitzung vom 13.1. $1931 \quad 55$

Sitzung vom 10. II. $1931 \quad 254$

- $\quad$ Sitzung vom 10. III. 1931405

Kölner Dermatologische Gesellschaft. Sitzungen vom 25. VII., 31. X. und

28. XL 193077

- $\quad$ Sitzungen vom 30.1, und 28. II. 1931181

Herbsttagung der Vereinigung rheinisch-westfälischer Dermatologen in

Elberfeld am 12. X. $1930 \quad 80$

Royal Society of Medicine. Section of Dermatology. Sitzungen vom 15. V., 19. VI. und 16. X. 1930 95

North of England Dermatological Society. Sitzungen vom 9. V. 1930 in

Liverpool und 27. VI. 1930 in Leeds 104

Société française de Dermatologie et de Syphiligraphie. Sitzung vom

18. IV. 1929107

Periodische Literatur. Zusammengestellt von Dr. Arthur Alexander-

Charlottenburg $\quad 114,182,268,420$

Buchbesprechungen 133, 217, 294, 437

Zur 25jährîgen Wiederkehr des Todestages von Fritz Schaudinn. Von Erich

Hoffmann-Bonn 296

Gedenkfeier zur 25. Wiederkehr des Todestages von Fritz Schaudinn im

Zoologischen Institut der Universität Berlin. Von Erich Hoffmann 438

Personalien und Tagesnachrichten 141, 220, 297, 440

Sachregister 441

Namenregister 449 